\title{
ORIENTED GRAPHITE SINGLE-CRYSTAL INCLUSIONS IN DIAMOND
}

\author{
Jürgen Glinnemann ${ }^{1 *}$, Katsuhiro Kusaka ${ }^{2}$, Jeff W. Harris ${ }^{3}$, Bernd Bleisteiner ${ }^{4}$ and Björn Winkler ${ }^{1}$ \\ ${ }^{1}$ University of Frankfurt/Main, Germany; ${ }^{2}$ High Energy Accelerator Research Organization (KEK), Japan; \\ ${ }^{3}$ University of Glasgow, UK; ${ }^{4}$ Jobin Yvon Raman Division, Germany
}

\section{OPTICAL MICROSCOPY}

Graphite is a rather abundant inclusion in natural diamond and occurs mostly as sheet-like masses parallel to any of the diamond octahedral $\{111\}$ planes and at any location inside its host (Harris, 1972; Harris and Vance, 1972).

In contrast to this, all but one of our 14 so far investigated specimens consist of an isolated centred graphite single crystal of linear extensions up to $300 \mu \mathrm{m}$. All diamonds are colourless, clear octahedra with rounded edges of 2 to $3 \mathrm{~mm}$ lengths, similar to those shown in Fig. 1, and weigh between 23 and $26 \mathrm{mg}$. They are derived from the Panda kimberlite (Carlson et al., 1999) in the Ekati Diamond Mine, Canada, and collected by one of us (J. W. Harris).

The inclusions exhibit typical euhedral graphite crystal forms, i.e. hexagonal lamellae (11 specimens) or hexag- onal dipyramids with pinacoids (2 specimens). Optical inspection revealed the graphite inclusions to lie with their (001) faces parallel to (111) faces of their diamond hosts (Fig. 1).

A 14th specimen (PAG02) is rather different from the others just described. The diamond exhibits in its centre a large (about $300 \mu \mathrm{m}$ edge length) region with a sharp hexagonal borderline filled with a "patchwork" of tiny graphite "islands" (Fig. 2). Several parallel lamellae up to approximately $5 \mu \mathrm{m}$ thickness can be distinguished, resulting in a hexagonal pyramidal form (Fig. 2, right). These special features suggest a "dissolution" of a graphite seed in a topotactic reaction with a growing diamond. In that case this graphite inclusion would be protogenetic with respect to its diamond host. The lamellae are approximately parallel to (not developed) (110) faces of diamond.
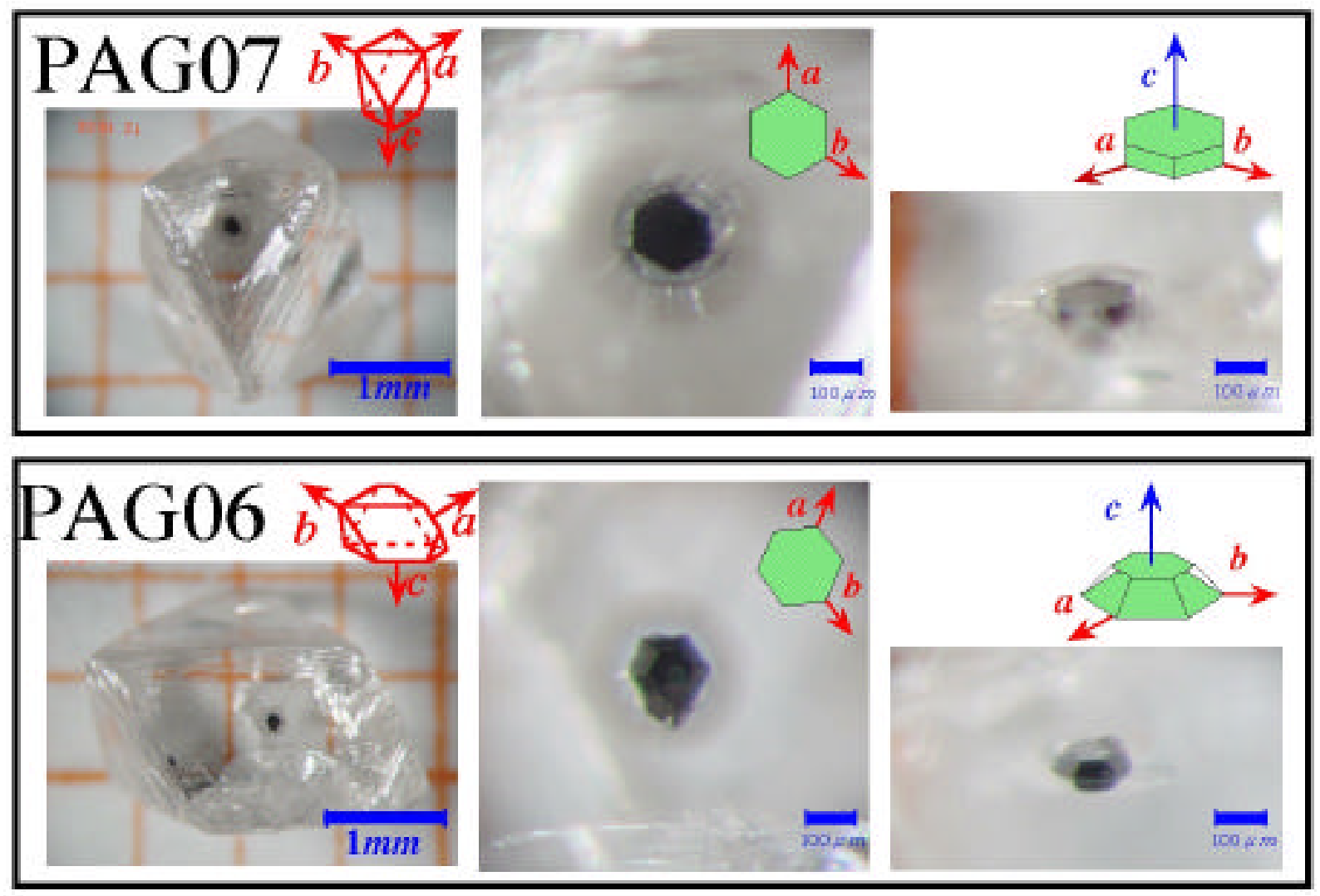

Figure 1: Two colourless, clear, octahedral diamonds with a single graphite inclusion. Left: complete specimens; middle: inclusions magnified, same view as left; right: inclined view. Arrows $\boldsymbol{a}, \boldsymbol{b}, \boldsymbol{c}$ in left indicate the directions of the diamond cubic crystallographic axes, arrows $\boldsymbol{a}, \boldsymbol{b}, \boldsymbol{c}$ in middle and right indicate the directions of the graphite hexagonal crystallographic axes. 


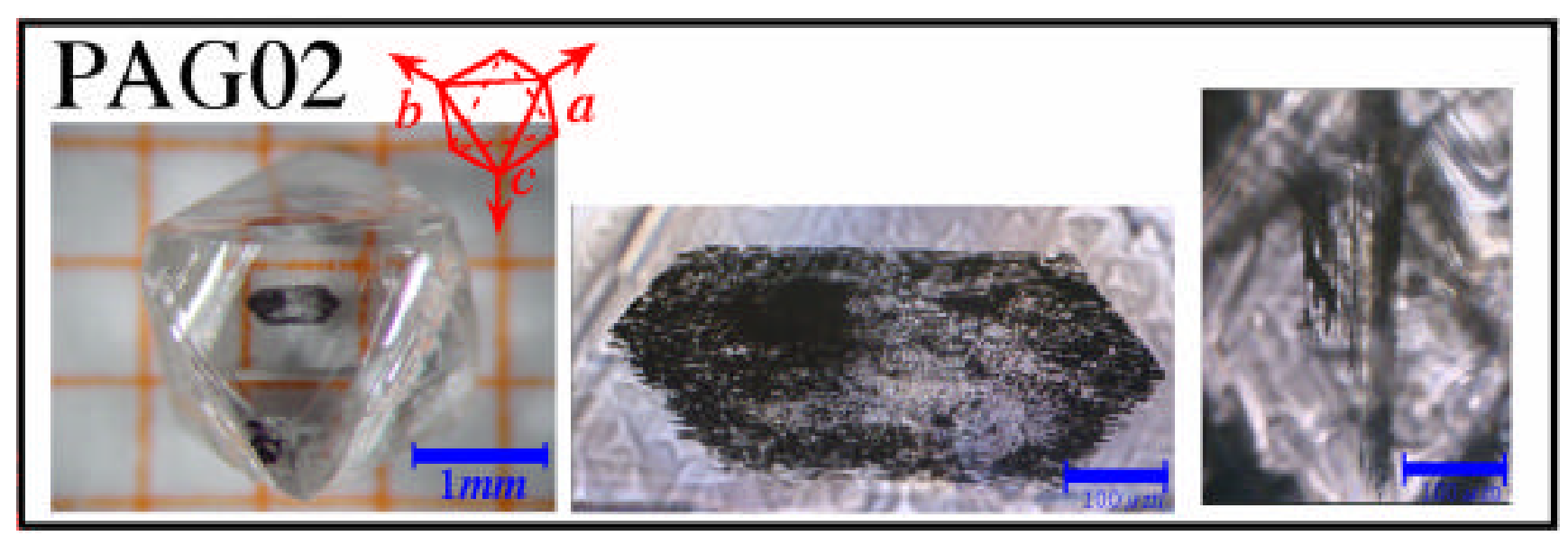

Figure. 2: Left: Specimen PAG02 with its large, thin inclusion exhibiting a sharp hexagonal borderline; middle: inclusion magnified to highlight the ,patchwork“ of graphite. Right: sideview; several thin, adjacant graphite sheets combine to a hexagonal pyramidal form.

\section{X-RAY SINGLE-CRYSTAL DIFFRACTION}

\section{Mutual ORIENTATION OF Diamond HOSt AND GRAPHITE INCLUSION}

In-house classical Laue-diffraction experiments confirm the optical observations on the euhedral graphite inclusions (13 specimens): they are well crystallized single crystals, as judged from sharp X-ray reflections (Fig. 3).

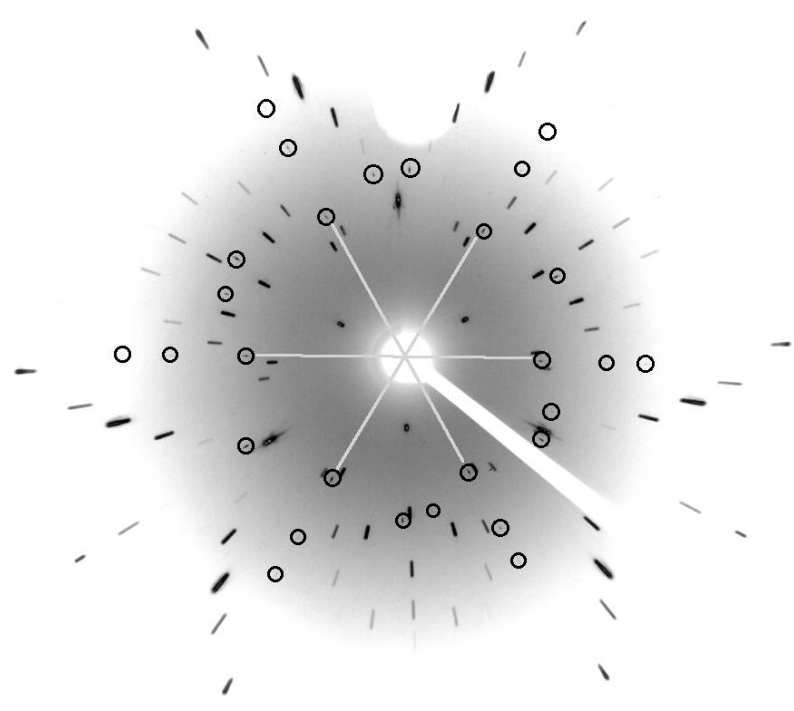

Figure 3: Example (specimen PAG07) of an X-ray Lauediffraction pattern. Incident X-ray along one of the $\langle 111\rangle$ directions of diamond. Large, intense reflections belong to the diamond host. Circled, faint, sharp reflections stem from the graphite inclusion. The graphite hexagonal axis [001] deviates by about $1.5^{\circ}$ from the diamond [111] direction. (Mo-radiation, $50 \mathrm{kV}, 40 \mathrm{~mA} ; 40 \mathrm{~mm}$ crystal-to-film distance, arbitrarily scaled here; diameter of primary X-ray beam $0.5 \mathrm{~mm}$.)
The mutual crystallographic orientation of diamond host and graphite inclusion in each specimen was computed from a number of diffraction angles of both phases on a single crystal diffractometer (CAD 4, Enraf Nonius). A summary is provided in Table 1 . The orientations clearly are regular. A detailed discussion of these results along the concept of 'coincidence site lattices (CSL)' is given by Glinnemann et al. (2003).

The X-ray investigations show these 13 graphite inclusions to be hexagonal, space group $P 6_{3} / m m c$ (no. 194).

Table 1: Experimental angles between selected lattice directions of graphite inclusions and their diamond hosts

\begin{tabular}{|c|c|c|}
\hline Specimen & Angle/ ${ }^{\circ}$ & Angle/ ${ }^{\circ}$ \\
\hline PAGno. & $\mathrm{G}[001] / \mathrm{D}<111>$ & $\mathrm{G}<100>/ \mathrm{D}<110>$ \\
\hline 01 & $4.3(6)$ & $32.7(2)$ \\
\hline 07 & $1.4(4)$ & $33.1(2)$ \\
\hline 10 & $0.0(5)$ & $35.3(1)$ \\
\hline 14 & $1.4(4)$ & $34.7(2)$ \\
\hline 15 & $0.2(6)$ & $37.0(2)$ \\
\hline 18 & $0.1(5)$ & $34.3(2)$ \\
\hline 12 & $0.2(6)$ & $15.6(5)$ \\
\hline 03 & $0.0(6)$ & $3.5(1)$ \\
\hline 06 & $0.0(4)$ & $3.3(1)$ \\
\hline 13 & $4.0(5)$ & $4.0(1)$ \\
\hline 17 & $0.0(4)$ & $4.4(1)$ \\
\hline \multirow[t]{3}{*}{19} & $2.7(6)$ & $5.4(1)$ \\
\hline & Angle/ ${ }^{\circ}$ & Angle/ ${ }^{\circ}$ \\
\hline & $\mathrm{G}[001] / \mathrm{D}<100>$ & $\mathrm{G}<100>/ \mathrm{D}<110>$ \\
\hline 16 & $6.0(4)$ & $0.4(1)$ \\
\hline
\end{tabular}


From the 14th specimen (PAG02, Fig. 2) only a broad 002 reflection (most intense reflection of the hexagonal graphite crystal structure) could be obtained so far. However, its position confirms the optical finding (Fig. 2, left), that graphite (001) is approximately parallel to a (110) internal face of diamond in this specimen.

\section{FoSSILIZED PRESSURE ON GRAPHITE INCLUSIONS IN DIAMOND}

The graphite crystal structure is exceptional anisotropic. Its hexagonal atomic layers parallel (001) are mutually only loosely bonded. An isostatic pressure of up to 3.5 GPa causes no significant compression of the $a$ lattice parameter, but the $c / c_{0}$ ratio is already reduced to 0.94 (Zhao and Spain, 1989).

\section{Table 2: Experimental lattice parameters of graphite inclusions in diamonds and estimated remnant pressures}

\begin{tabular}{lccccc}
\hline $\begin{array}{l}\text { Sample } \\
\text { PAG } \\
n o .\end{array}$ & $a_{0} / \AA$ & $\begin{array}{c}\text { Lattice } \\
a / a_{0}\end{array}$ & $\begin{array}{c}\text { parameters } \\
c_{0} / \AA\end{array}$ & $c / c_{0}$ & $\begin{array}{c}P \\
\mathrm{GPa}\end{array}$ \\
\hline 01 & $2.457(1)$ & 0.998 & $6.569(3)$ & 0.980 & 1.1 \\
03 & $2.456(1)$ & 0.998 & $6.570(2)$ & 0.980 & 1.1 \\
06 & $2.455(1)$ & 0.997 & $6.392(1)$ & 0.954 & 2.6 \\
07 & $2.458(1)$ & 0.998 & $6.411(1)$ & 0.957 & 2.4 \\
10 & $2.459(1)$ & 0.999 & $6.423(1)$ & 0.959 & 2.3 \\
12 & $2.459(1)$ & 0.999 & $6.447(1)$ & 0.962 & 2.1 \\
13 & $2.453(1)$ & 0.997 & $6.448(1)$ & 0.962 & 2.1 \\
14 & $2.457(1)$ & 0.998 & $6.557(2)$ & 0.978 & 1.2 \\
15 & $2.452(1)$ & 0.996 & $6.552(1)$ & 0.978 & 1.2 \\
16 & $2.454(1)$ & 0.997 & $6.443(1)$ & 0.961 & 2.1 \\
17 & $2.456(1)$ & 0.998 & $6.508(1)$ & 0.971 & 1.6 \\
18 & $2.455(1)$ & 0.997 & $6.580(1)$ & 0.982 & 1.0 \\
19 & $2.458(1)$ & 0.998 & $6.696(1)$ & 0.999 & 0.1 \\
\hline
\end{tabular}

Lattice parameters of hexagonal graphite at ambient conditions: $a_{0}=2.462(1) \AA, c_{0}=6.701(2) \AA$ (Zhao and Spain, 1989).

In relation to ambient conditions $\left(a_{0}, c_{0}\right)$, our in situ measurements of the lattice parameters of graphite inclusions also reveal only a significant shortening of $c$ (Table 2). Assuming isostasy, a comparison of our experimental $c$-values with those of Zhao and Spain (1989) shows residual pressures between 1.0 and 2.6 GPa (Table 2). Specimen PAG19 is not representative in this respect, because the graphite is located close to an outer diamond (111) fracture face. No correlation between size, crystal shape or orientation of a graphite inclusion with the measured remnant pressure and size of the diamond host is observed.

The estimated fossilized pressures agree well with those on other inclusions in diamond. For example, Sobolev et al. (2000) report remnant pressures of about $3.6 \mathrm{GPa}$ for coesite inclusions.

\section{MICRO-RAMAN SPECTROSCOPY}

The spectra given in Fig. 4 were obtained on specimen PAG07 with a LabRAM system (Jobin Yvon), 100x objective lens, $1 \mu \mathrm{m}$ confocal laser spot, $633 \mathrm{~nm}$ laser excitation wavelength, and a HeNe laser power less than $1 \mathrm{~mW}$. The excited laser beam was along that diamond [111] direction which is approximately parallel to the graphite [001] direction (Table 1). So far, only a qualitative analysis of these spectra can be given.

Due to the high refractive index of diamond (about 2.41 for the wavelength used) the original (in air) laser spot of $1 \mu \mathrm{m}$ will distinctly broaden with depth of penetration into the diamond. To hit the surface of the graphite inclusion in specimen PAG07, this path length may be up to $0.6 \mathrm{~mm}$, thus a broadening of the spot to some tens of micrometers has to be taken into account. Also the focus will move and elongate with depth, both in the order of magnitude of 100 to $200 \mu \mathrm{m}$.

We took the appearance of maxima of the two graphite signals at 1593 and $2722 \mathrm{~cm}^{-1}$ as the position of highest laser intensity on the graphite surface (Fig. 4, second spectrum from bottom). The spectrum at the bottom was obtained by moving the sample further $20 \mu \mathrm{m}$ against the objective. The opaqueness of graphite forced the signals to weaken and nearly disappear at this position. At $20 \mu \mathrm{m}$ 'above' the graphite surface (third spectrum from bottom) the graphite signals are also already significantly reduced, but they are still observed even in the uppermost spectrum, after a $100 \mu \mathrm{m}$ move of the sample away from the objective. The diamond signal at $2470 \mathrm{~cm}^{-1}$ is too faint to appear in the spectrum with the most intense graphite lines (second from bottom), but rises with distance from graphite.

With increasing distance of the objective from the graphite inclusion severe fluorescence and a broad band around $3100 \mathrm{~cm}^{-1}$ arise. A decision about the nature of these features and whether they are linked to the graphite/diamond interface or to the bulk of the diamond host deserves further investigations. Remarkably, the broad signal around $3100 \mathrm{~cm}^{-1}$ covers the range of $\mathrm{C}-\mathrm{H}$ stretching modes in methane under pressure (Wu et al., 1995) and of similar modes of optical centres caused by hydrogen atoms in diamond (Wilks and Wilks, 1991). 


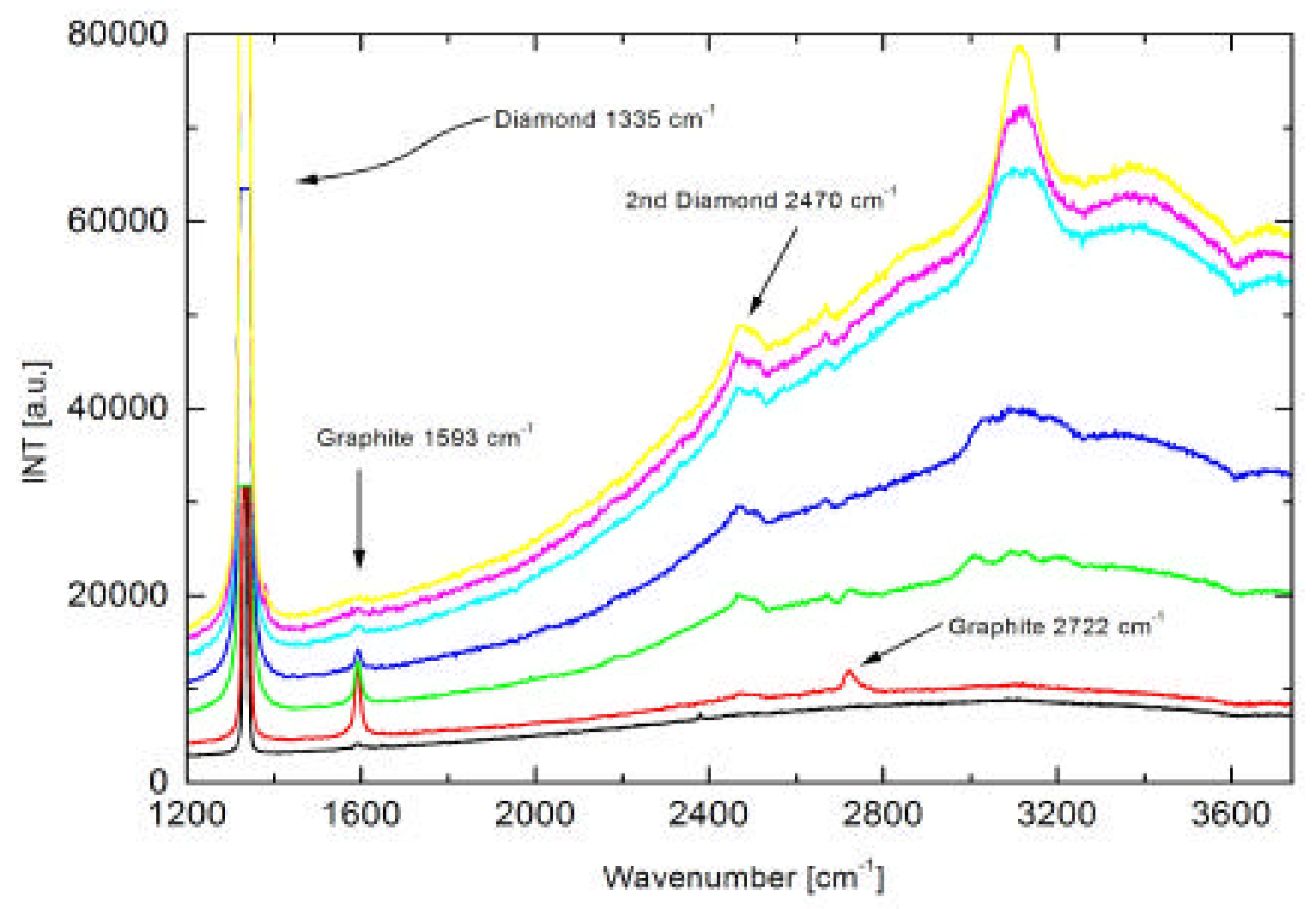

Figure 4: Micro-Raman spectra of specimen PAG07. The second line from bottom (red) shows most intense signals from graphite. The signals above this spectrum are obtained after stepwise increase of the distance between sample and objective lens by $20 \mu \mathrm{m}$. For the spectrum at the bottom the distance was reduced by $20 \mu \mathrm{m}$ with respect to that for the second spectrum from bottom. The origin of the broad band around $3100 \mathrm{~cm}^{-1}$ and the increase in fluorescence with the distance from the graphite deserve further investigation.

Acknowledgement: J. G. is grateful for financial support from the German Science Foundation (DFG) within project Wi $1232 / 18-1$.

\section{REFERENCES}

Carlson, J. A., Kirkley, M. B., Thomas, E. M., Hillier, W. D., 1999. Recent Canadian kimberlite discoveries. Proc. VIIth Int. Kimberlite Conf. Red Roof Design, Cape Town, South Africa, pp. $81-89$.

Glinnemann, J., Kusaka, K., Harris, J.W., 2003. Oriented graphite single crystal inclusions in diamond. Zeitschr. Kristallogr., accepted.

Harris, J.W., 1972. Black material on mineral inclusions and in internal fracture planes in diamond. Contr. Mineral. and Petrol. 35, $22-33$.

Harris, J.W., Vance, E.R., 1972. Induced graphitisation around crystalline inclusions in diamond. Contr. Mineral. and Petrol. 35, $227-234$.
Sobolev, N.V., Fursenko, B.A., Goryainov, S.V., Shu, J., Hemley, R.J., Mao, H., 2000. Fossilized high pressure from the Earth's deep interior: The coesite-in-diamond barometer. Proc. Natl. Acad. Sci. USA 97(22), 11875 - 11879. (Available free of charge: www.pnas.org/cgi/doi/ 10.1073/pnas.220408697).

Wilks, J., Wilks, E., 1991. Properties and Applications of Diamond. Butterworth-Heinemann, Oxford, pp. $91-92$.

Wu, Y.H., Sasaki, S., Shimizu, H., 1995. High-pressure Raman study of dense methane: $\mathrm{CH} 4$ and CD4. J. Raman Spectrosc. 26, $963-967$.

Zhao, Y.X., Spain, I.L., 1989. X-ray diffraction data for graphite to $20 \mathrm{GPa}$. Phys. Rev. B 40, 993 - 997.

Contact: J Glinnemann, Inst. of Mineralogy/Crystallography,

Univ. of Frankfurt/Main, Senckenberganlage 30,

D - 60054 Frankfurt/Main

E-mail: Glinnemann@kristall.uni-frankfurt.de 